\title{
The degradation of phenol derivates from wastewaters by electrochemical treatment
}

\author{
A. Bebeselea ${ }^{1}$, F. Manea ${ }^{1}$, C. Radovan ${ }^{2}$, G. Burtica ${ }^{1}$, \\ C. Teodosiu ${ }^{3}$, A. Pop ${ }^{1}$, C. Proca ${ }^{1} \&$ I. Corb ${ }^{1}$ \\ ${ }^{I}$ Politehnica University of Timisoara, Timisoara, Romania \\ ${ }^{2}$ West University of Timisoara, Timisoara, Romania \\ ${ }^{3}$ Gh.Asachi" Technical University of Iasi, Iasi, Romania
}

\begin{abstract}
A group of carbon-based electrodes, i.e., glassy-carbon (GC), boron doped diamond (BDD), expanded graphite-polystyrene composite (EG-PS), expanded graphite-epoxy composite (EG-Epoxy) and expanded graphite-carbon nanofiberepoxy composite (EG-CNF-Epoxy) electrodes were investigated for electrochemical degradation of 4-chlorophenol (4-CP). Based on the investigated electrochemical techniques, e.g., cyclic voltammetry (CV) and chronoamperometry (CA), the operation conditions and the electrode suitable for electrochemical oxidation of 4-CP were selected. In addition, multiple pulsed amperometry (MPA) was applied for the in-situ electrochemical cleaning of the electrode surface to improve the electrode efficiency. The BDD electrode exhibited the best features related to the 4-CP electrochemical degradation efficiency.
\end{abstract}

Keywords: wastewater, electrochemical degradation, 4-CP, carbon-based anodes.

\section{Introduction}

The phenol derivates are toxic and non-biodegradable organic compounds included in the list of priority phenols, which are used/produced in several industrial processes, being commonly used as preservatives, disinfectants, in pulp processing, in the manufacture of pesticides and intermediars [1-4].

The choice of wastewater treatment method depends on the treatment performance and economic component. Due to the treatment of industrial 
effluents requiring costly physical or physicochemical pretreatment, electrochemical is becoming a new alternative for the wastewater treatment and is replacing the traditional processes. The electrochemical wastewater treatment technology can be regarded as environmentally compatible, versatile, safe, because the electrons play the role of a clean reagent, without additional chemicals [5-8].

The practical application of electrochemical oxidation processes to the destruction of organic pollutants in water solution requires the essential features of the anode related to the oxidation process efficiency [9]. Function of the anode type, organic pollutants can be destroyed electrochemically by direct anodic oxidation (DSA type) or by an indirect oxidation process that involves the electrochemical generation of powerful oxidants as hydroxyl radical, peroxodisulfuric acid, etc. [10].

In the electrochemical processes, the aim is the complete oxidation of organics to $\mathrm{CO}_{2}$ or the conversion of the toxic organics to biocompatible compounds [1]. It has been reported [2,3] that the phenol electrooxidation produces electrode fouling by electropolimerization that leads to a low rate of oxidation, low permeability and strong adhesion on the electrode. On the other hand, it was shown that for removal of some phenolic compounds from wastewaters, a rather effective way is the adsorption of these compounds in the form of oligomeric and polymeric compounds on a high-surface-area carbon electrode [5].

The objective of the present study is to give an overall comparison of the glassy-carbon (GC), boron doped diamond (BDD), expanded graphite-epoxy composite (EG-Epoxy), expanded graphite-polystyrene composite (EG-PS), expanded graphite-carbon nanofiber-epoxy composite (EG-CNF-Epoxy) electrodes. The cyclic voltammetric behavior was investigated first to reveal the basic electrochemical performance difference. 4-chlorophenol (4-CP) was used as model pollutants to compare the activity of the electrodes under the same conditions by chronamperometry (CA). In order to improve the electrode activity by electrochemical cleaning during the electrochemical treatment, multiple pulsed amperometry (MPA) technique was tested. The results obtained will help to select appropriate electrodes for the anodic oxidation of pollutants.

\section{Experimental}

The composite electrodes were prepared from low viscosity polystyrene pellets (PS N2000, Crystal from Shell) or two-component epoxy resin (LY5052, Araldite) mixed with two types of conductive carbon fillers: expanded graphite (EG) powder (Conductograph, SGL Carbon) and carbon nanofibers (CNF) (PS447 BOX). In the case of the EG-Epoxy composite the full amount of EG to the matrix resin was not added directly, due to the high surface area of the graphite flakes, therefore, the mixing was performed in a roll-mill at room temperature. The two parts of the epoxy were mixed together and the full amount of the EG was added in steps forming a thick paste. Then the epoxy was cured in a hot press at $80{ }^{\circ} \mathrm{C}$ for 40 minutes. Simultaneously the material was shaped in a plate 
of $1 \mathrm{~mm}$ thickness. The plate was slowly cooled down (for about $12 \mathrm{~h}$ ) to the room temperature without removing the applied pressure. The graphite filler EG was mixed with the PS N2000 matrix in a roll-mill at $250{ }^{\circ} \mathrm{C}$ for about $30 \mathrm{~min}$. A plate of approximately $1 \mathrm{~mm}$ was pressed by a hot press (Fontaijne Holland) at the same temperature.

For CNF-EG-Epoxy composite, CNF and EG have been mixed with the two components of the epoxy in a roll-mill at room temperature and the obtained paste was cured in a hot press at $80{ }^{\circ} \mathrm{C}$ for 40 minutes. Simultaneously the material was shaped in a plate of approximately $1 \mathrm{~mm}$ thickness. The plate was slowly cooled down (for about $12 \mathrm{~h}$ ) to the room temperature without removing the applied pressure.

Plates with a surface area of $4 \mathrm{~cm}^{2}$ were cut from all compositions put on a glass supports and electrical contacts were made using a silver paint. The electrodes were isolated on the sides by epoxy resin. Prior to use, this working electrode was gradually cleaned, first polished with abrasive paper and then on a felt-polishing pad by using $0.03 \mu \mathrm{m}$ alumina powder (Metrohm, Switzerland) in distilled water for 5 minutes and rinsing with distilled water. The cylindrical glassy carbon (GC) and boron-doped diamond (BDD) electrodes with the surface areas of $2.81 \mathrm{~cm}^{2}$ and respective, $0.07068 \mathrm{~cm}^{2}$ were commercial types, provided by Metrohm and respective, Windsor Scientific Ltd, UK.

The electrochemical performances of these electrodes were studied by cyclic voltammetry $(\mathrm{CV})$, chronoamperometry (CA) and multiple-pulsed amperometry (MPA). Subsequently, an electrochemical pretreatment by three repetitive cyclings between $-0.5 \mathrm{~V}$ to $1.25 \mathrm{~V}$ vs. $\mathrm{SCE}$ in $0.1 \mathrm{M} \mathrm{Na}_{2} \mathrm{SO}_{4}$ supporting electrolyte was performed. All measurements were carried out using an Autolab potentiostat/galvanostat PGSTAT 302 (Eco Chemie, The Netherlands) controlled with GPES 4.9 software and a three-electrode cell, with a saturated calomel electrode as reference electrode, a platinum counter electrode and a carbon-based composite, GC or BDD as working electrode.

The concentration of 4-CP before and after the degradation process was determined using a UV-VIS spectrometer by type Jasco V-530, at the wavelength of $226 \mathrm{~nm}$.

4-chlorophenol and sodium sulphate were analytical grade from Merck, and the solutions were freshly prepared with double-distilled water.

\section{Results and discussion}

Figures 1 a-e show first cyclic voltammograms (CVs) at scan rate of $0.05 \mathrm{Vs}^{-1}$ of GC, BDD, EG-PS composite, EG-Epoxy composite and CNF-EG-Epoxy composite electrodes in $0.1 \mathrm{M} \mathrm{Na}_{2} \mathrm{SO}_{4}+0.2 \mathrm{mM}$ 4-CP. The voltammetric parameters of the oxidation of 4-CP at all carbon based material electrodes are gathered in table 1 .

It can be noticed for each electrode the potential value at which the oxidation process occurred, and the lowest potential value was recorded for BDD electrode. Starting with second CV the peak current decreased by quite $100 \%$ for CNF-EG-Epoxy composite electrode, which is due to the electrode fouling. 


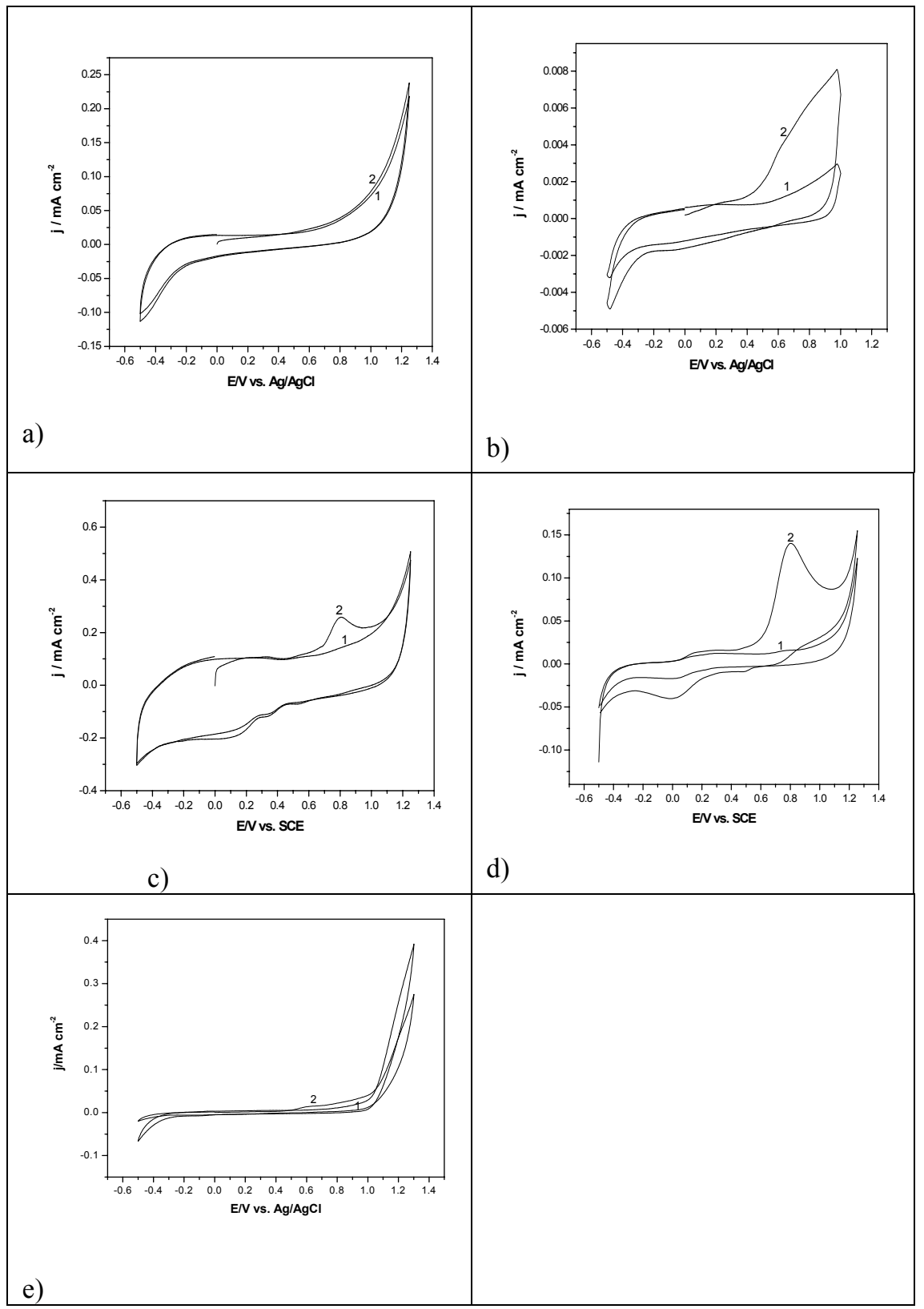

Figure 1: Cyclic voltammograms in $0.1 \mathrm{M} \mathrm{Na}_{2} \mathrm{SO}_{4}$ supporting electrolyte and in the presence of $0.2 \mathrm{mM} 4-\mathrm{CP}$ of: a)-GC, b)-BDD, c)-EGPS, d)-EG -Epoxy, e)- EG-CNF-Epoxy electrodes; starting potential: $0 \mathrm{~V}$ vs. SCE; potential range: $-0.5 \mathrm{~V} \rightarrow+1.25 \mathrm{~V} / 1 \mathrm{~V} \rightarrow-$ $0.5 \mathrm{~V}$ vs. SCE; scan rate: $0.05 \mathrm{~V} \cdot \mathrm{s}^{-1}$. 
Table 1: $\quad$ Voltammetric parameters of the oxidation of 4-CP for several carbon based material electrodes.

\begin{tabular}{|l|c|c|c|}
\hline $\begin{array}{c}\text { Material } \\
\text { electrodes }\end{array}$ & Scan number & $\mathrm{j}^{*}\left(\mathrm{mAcm}^{-2}\right)$ & $\mathrm{E}(\mathrm{V})$ \\
\hline GC & 1 & $2.88510^{-3}$ & 0.8 \\
& 2 & $2.47710^{-3}$ & 0.8 \\
BDD & 3 & $2.06810^{-3}$ & 0.8 \\
& 1 & $2,6310^{-3}$ & 0.6 \\
EG-PS & 2 & $9,4210^{-4}$ & 0.6 \\
& 3 & $8,6410^{-4}$ & 0.6 \\
EG-Epoxy & 2 & 0.115347 & 0.8 \\
& 3 & 0.043995 & 0.8 \\
\multirow{2}{*}{ CNF-EG-Epoxy } & 1 & $3.63310^{-3}$ & 0.8 \\
& 2 & - & 0.8 \\
& 3 & - & 0.8 \\
& 2 & $8,20310^{-3}$ & 0.8 \\
& 3 & - & 0.8 \\
& 2 & - & 0.8 \\
\hline
\end{tabular}

*The capacitive component has been subtracted from the current densities.

For GC, BDD and EG-PS composite, EG-Epoxy composite electrodes the peak current decreases by $64.18 \%, 13.10 \%, 58.80 \%$, and respective $54.64 \%$, starting with the second CV. In general, the electrode fouling is owing the complex mechanism of phenols oxidation on carbon based electrode, which involves both the adsorption of reactant/intermediate or final oxidation products and the formation of passive, nonconductive layer of oligomer products of oxidation process on its surface $[3,5,11,12]$.

The effect of initial concentration of 4-CP on the oxidation process for both potential ranges of water decomposition and stabilization, and expressed by the cyclic scanning is shown as example for BDD electrode in Figure 2. It is obvious that increasing initial concentration decreased the rate of 4-CP oxidation due to faster electrode fouling. On the other hand, it is known that increasing the bulk solution concentration should increase the rate of 4-CP oxidation if the process is mass transfer controlled [12]. From Figure 2 it can be see that no significant electrode fouling occurred at BDD electrode for the oxidation of $1 \mathrm{mM} 4-\mathrm{CP}$ under both potential ranges of water decomposition and stabilization.

The effect of the scan rate on the anodic peak current of the first CV of BDD electrode tested in $0.1 \mathrm{M} \mathrm{Na}_{2} \mathrm{SO}_{4}+0.2 \mathrm{mM} 4-\mathrm{CP}$ is shown in Figure 3. This experiment was performed for each electrode but not all results are presented. The electrode cleaning during CV running at different scan rates was performed. The linear proportionality of the anodic peak current with the square root of the scan rate obtained for all tested electrodes indicated control by mass transport. No intercept of 0 suggested that the adsorption steps and specific surface reaction cannot be neglected (insert a of Figure 3). The most strong adsorption 


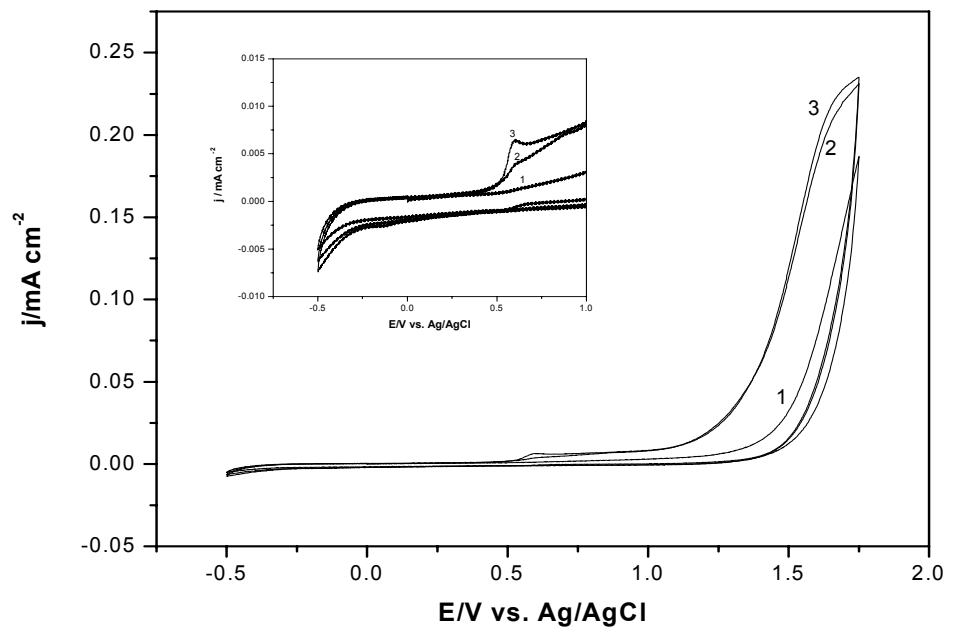

Figure 2: The first scanning of cyclic voltammograms of the BDD electrode in $0.1 \mathrm{M} \mathrm{Na}_{2} \mathrm{SO}_{4}$ solution (curve 1) with $0.2 \mathrm{mM} \mathrm{4-CP}$ (curve 2) and $1 \mathrm{mM} 4-\mathrm{CP}$ (curve 3 ): potential range: $-0.5 \mathrm{~V} \rightarrow+1.75 \mathrm{~V} \rightarrow-$ $0.5 \mathrm{~V}$ vs. $\mathrm{Ag} / \mathrm{AgCl}$; scan rate $=0.05 \mathrm{~V}^{*} \mathrm{~s}^{-1}$. Insert: details of the limited potential range.

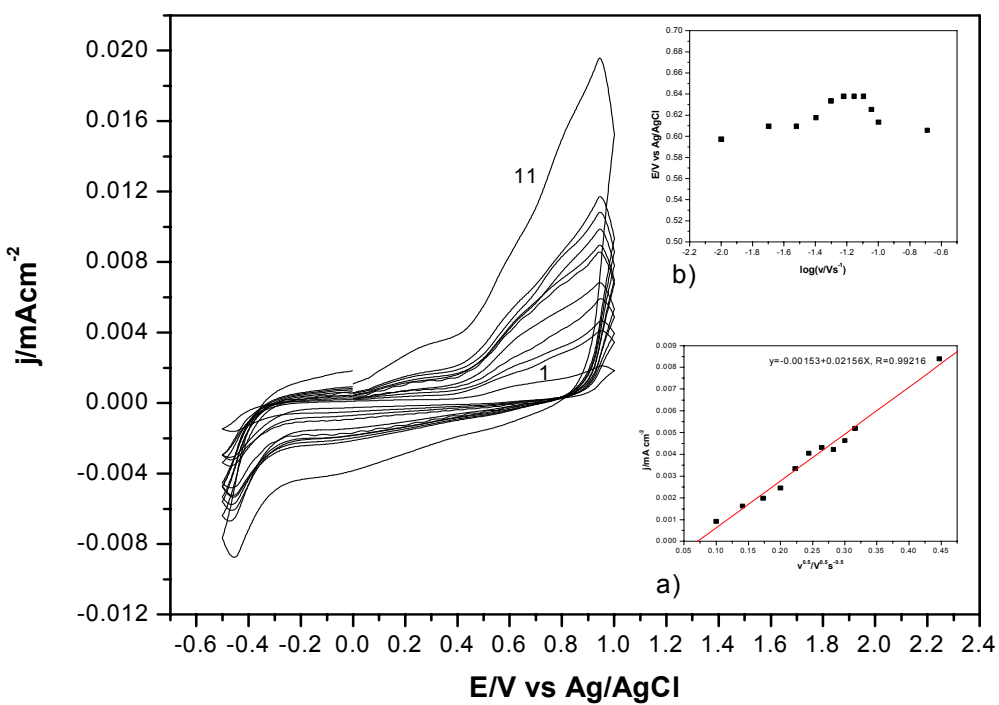

Figure 3: Cyclic voltammograms of the BDD electrode in $0.1 \mathrm{M} \mathrm{Na} \mathrm{SO}_{4}$ solution and $0.2 \mathrm{mM} \mathrm{4-CP}$ at different scan rates, curves 1-11: $0.01-0.2 \mathrm{Vs}^{-1}$. Insert: a)-Plot of the anodic peak current recorded at $0.6 \mathrm{~V}$ vs. $\mathrm{Ag} / \mathrm{AgCl}$ versus the square root of scan rate, b)-Plot of the anodic peak potential versus the logarithm of the scan rate. 
effects were noticed for EG-CNF-Epoxy electrode, these results being in concordance with those obtaining by three consecutive scanning using CV. Even the oxidation potential value is almost constant (inset b of Figure 3 ) the 4-CP oxidation is not reversible because no corresponding reduction cathodic peak appeared by backward scanning.

In order to obtain information about electrode fouling under the anodic potential range of oxygen evolution and of limiting current (1.1 and respective, $0.6 \mathrm{~V}$ vs. $\mathrm{Ag} / \mathrm{AgCl}$ ) chronoamperometric measurements were performed. The chronoamperograms recorded for each electrode during $2 \mathrm{~h}$ are presented in Figure 4.

The shapes of the chronoamperograms indicated GC electrode as most suitable for 4-CP oxidation under the potential range of oxygen evolution. However, it is necessary to take into account that for all electrodes, except BDD electrode the major process is oxygen evolution, which hamper the electrode fouling. Depending on the applied current density/potential, two different operating regimes have been identified $[3,5]$ i.e., $\mathrm{j}_{\text {appl }}<\mathrm{j}_{\text {lim }}$ the electrolysis is under current control with the current efficiency of $100 \%$ and $i_{\text {appl }}>i_{\text {lim }}$ the electrolysis is under mass-transport control and the secondary reactions of oxygen evolution are involved with a decreasing of current efficiency. Except $\mathrm{BDD}$, the electrode fouling occurred after a few minutes when $\mathrm{j}_{\text {appl }}<\mathrm{j}_{\text {lim }}$ (the results are not shown).

Figure 5 shows an example of multiple pulsed amperogram recorded for BDD electrode in $0.1 \mathrm{M} \mathrm{Na}_{2} \mathrm{SO}_{4}$ and in the presence of $0.2 \mathrm{mM} \mathrm{4-CP.} \mathrm{After} \mathrm{the} \mathrm{time}$ duration of 900 seconds, the electrode fouling started to occur.

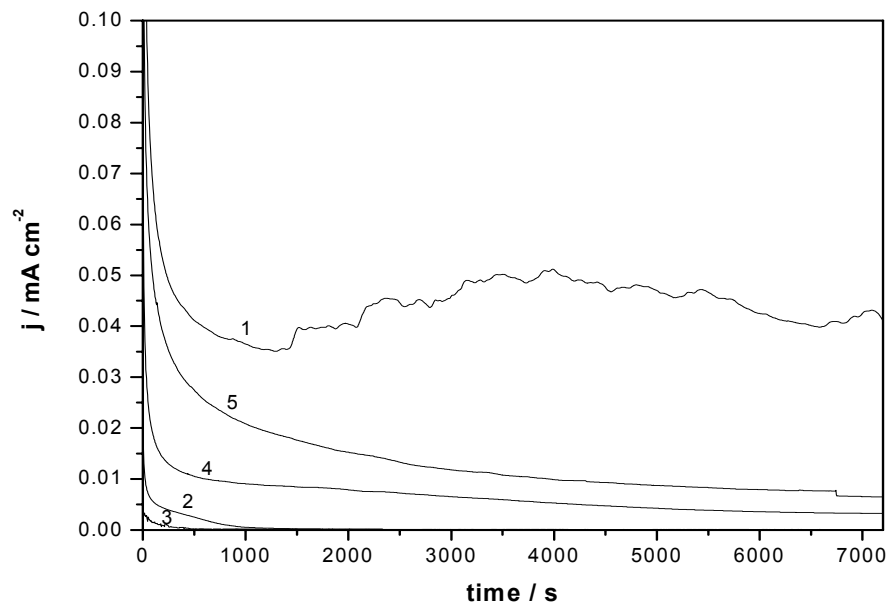

Figure 4: Chronoamperograms recorded at $+1.1 \mathrm{~V}$ vs. $\mathrm{Ag} / \mathrm{AgCl}$ for: $1-\mathrm{GC}$, 3-EG-PS, 4-EG-Epoxy, 5-CNF-EG-Epoxy electrodes, respective at $+0.6 \mathrm{~V}$ vs. $\mathrm{Ag} / \mathrm{AgCl}$ for-BDD electrode (curve 2), in $0.1 \mathrm{M}$ $\mathrm{Na}_{2} \mathrm{SO}_{4}$ supporting electrolyte and in the presence of $0.2 \mathrm{mM} 4$ CP. 


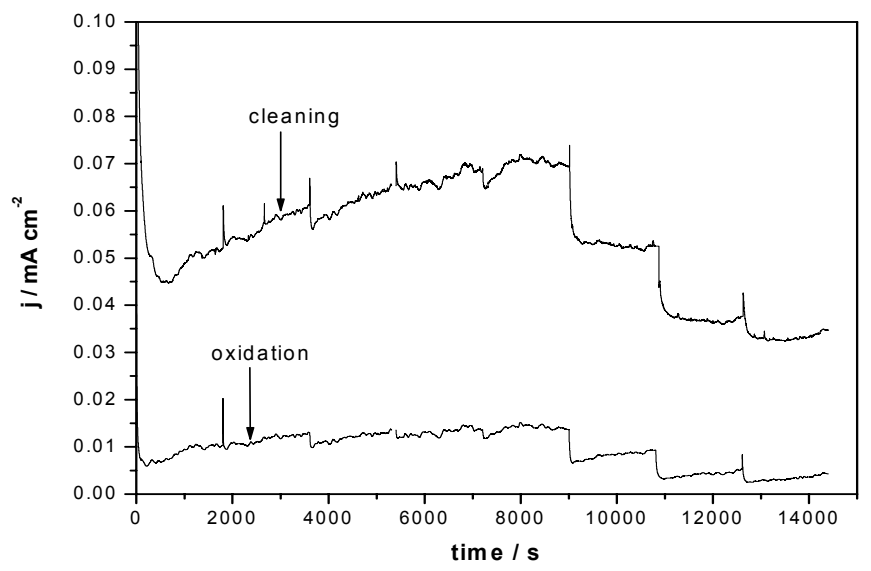

Figure 5: Multiple pulsed amperogram of the BDD electrode in $0.1 \mathrm{M}$ $\mathrm{Na}_{2} \mathrm{SO}_{4}$ supporting electrolyte and in the presence of $0.2 \mathrm{mM} 4-$ $\mathrm{CP}$; $\mathrm{E}_{\text {oxidation }}$ was $+0.6 \mathrm{~V}$ and $\mathrm{E}_{\text {cleaning }}$ was $+1.5 \mathrm{~V}$ vs. $\mathrm{Ag} / \mathrm{AgCl}$; $\mathrm{t}_{\text {oxidation }}$ was 0.05 seconds and $\mathrm{t}_{\text {cleaning }}$ was 0.2 seconds.

For all electrodes application in the 4-CP degradation by using both CA and MPA techniques for 2 hours, after each measurement the final concentration of 4-CP was determined spectrophotometrically and the electrochemical efficiency for 4-CP oxidation was obtained based on the equation [13]:

$$
E_{C P}=\frac{(C P 0-C P)}{C \cdot S} V\left(\mathrm{~g} / \mathrm{C} \cdot \mathrm{cm}^{2}\right)
$$

where $\mathrm{CP}_{0}-\mathrm{CP}$ is the change in the 4-CP concentration during chronoampeormetric test for a charge consumption of $\mathrm{C}$ corresponding to $2 \mathrm{~h}, \mathrm{~V}$ is the sample volume $(50 \mathrm{ml})$ and $\mathrm{S}$ is the area of the electrode $\left(\mathrm{cm}^{2}\right)$.

The electrochemical performance for 4-CP oxidation of the tested electrodes using CA and MPA techniques are gathered in table 2.

Table 2: $\quad$ The electrochemical efficiency $\left(\mathrm{E}_{\mathrm{CP}}\right)$ for the 4-CP degradation.

\begin{tabular}{|c|c|c|}
\hline Electrode material & $\mathrm{CA}-\mathrm{E}_{\mathrm{CP}}\left(\mathrm{g} \cdot \mathrm{C}^{-1} \cdot \mathrm{cm}^{-2}\right)$ & MPA-E $E_{\mathrm{CP}}\left(\mathrm{g} \cdot \mathrm{C}^{-1} \cdot \mathrm{cm}^{-2}\right)$ \\
\hline GC & $0.12 \cdot 10^{-4}$ & $0.45 \cdot 10^{-4}$ \\
\hline BDD & 67.30 & 70.70 \\
\hline EG-PS & $3.20 \cdot 10^{-2}$ & 0.86 \\
\hline EG-Epoxy & $4.74 \cdot 10^{-4}$ & $16.10 \cdot 10^{-4}$ \\
\hline CNF-EG-Epoxy & $2.73 \cdot 10^{-4}$ & $12.00 \cdot 10^{-3}$ \\
\hline
\end{tabular}

MPA technique improved the electrochemical performance for 4-CP oxidation for all tested electrodes. It is clear that BDD exhibited the best electrochemical activity for 4-CP oxidation. The electrochemical performances 
of the composite electrodes were better than conventional GC electrode but worse than BDD electrode.

\section{Conclusions}

The performances of GC, EG-Epoxy, EG-PS, EG-CNF-Epoxy and BDD electrodes were compared under the same conditions in order to apply them for the electrochemical degradation of 4-CP. Experimental results indicate that BDD was a much better anode than the other electrodes in terms of electrochemical activity. MPA application improved the electrochemical performance for all tested electrodes. The electrochemical efficiency for 4-CP degradation for the BDD anode was very high compared with other ones, and conventional GC electrode gave the worst results.

\section{Acknowledgements}

This research was supported by the Romanian National Research of Excellence Programs CEEX, RIWATECH - grant 62/03.10.2005, PROAQUA - grant 631/03.10.2005 and BIOCHEM - grant 59/03.10.2005.

\section{References}

[1] Zhi, J.F., Wang, H.B., Nakashima, T., Rao, T.N., Fujishima, A., Electrochemical incineration of organic pollutants on baron-doped diamond electrode. Evidence for direct electrochemical oxidation pathway. J. Phys. Chem., B107, pp. 13389-13395, 2003.

[2] Ezerskis, Z. and Jusys, Z., Oxidation of chlorophenols on Pt electrode in alkaline solution studied by cyclic voltammetry, galvanostatic electrolysis, and gas chromatography-mass spectrometry. Pure Appl. Chem., 73(12), pp. 1929-1940, 2001.

[3] Gherardini, F., Michaud, P.A., Panizza, M., Comninellis, C., Vatistas, N., Electrochemical oxidation of 4-chlorophenol for wastewater treatment. Definition of normalized current efficiency $(\Phi)$. J. Electrochem. Soc., 148(6), pp. D72-D82, 2001.

[4] Berrios, C., Arce, R., Rezende, M.C., Ureta-Zanartu, M.S., Gutierrez, C., Electrooxidation of chlorophenol at a glassy carbon electrode in a $\mathrm{pH} 11$ buffer. Electrochimica Acta, 53, pp. 2768-2775, 2008.

[5] Skowronski, J.M. and Krawczyk, P., Improved electrooxidation of phenol at exfoliated graphite electrodes. J. Solid State Electrochem., 11, pp. 223 230, 2007.

[6] Chen, X., Gao, F., Chen, G., Comparison of $\mathrm{Ti} / \mathrm{SnO}_{2}-\mathrm{Sb}_{2} \mathrm{O}_{5}$ electrodes for pollutant oxidation. Journal of Applied Electrochemistry, 35, pp. 185-191, 2005.

[7] Canizares, P., Lobato, J., Paz, R., Rodrigo, M.A., Saez, C., Electrochemical oxidation of phenolic wastewaters with baron-doped diamond anodes. Water Research, 39, pp. 2687-2703, 2005. 
[8] Zaggout, F.R., Ghalwa, N.A., Removal of o-nitrophenol from water by electrochemical degradation using a lead oxide/titanium modified electrode. Journal of Environmental Management, 86, pp. 291-296, 2008.

[9] Canizares, P., Saez, C., Lobato, J., Rodrigo, M.A., Detoxification of synthetic industrial wastewaters using electrochemical oxidation with baron-doped diamond anodes. Journal of Chemical Technology and Biotechnology, 81, pp. 352-358, 2006.

[10] Codognoto, L., Machado, S.A.S., Avaca, L.A., Electrochemical determination and removal of pentachlorophenol at diamond electrodes. Portugaliae Electrochimica Acta, 23, pp. 225-246, 2005.

[11] Azzam, O.M., Al-Tarazi, M., Tahboub, Y., Anodic destruction of 4chlorophenol solution. Journal of Hazardous Materials, B75, pp. 99-113, 2000.

[12] Ureta-Zanartu, M.S., Bustos, P., Berrios, C., Diez, M.C., Mora, M.L., Gutierrez, C., Electrooxidation of 2,4-dichlorophenol and other polychlorinated phenols at a glassy carbon electrode. Electrochimica Acta, 47, pp. 2399-2406, 2002.

[13] Wang, Y.H., Chan, K.Y., Li, X.Y., So, S.K., Electrochemical degradation of 4-chlorophenol at nickel-antimony doped tin oxide electrode. Chemosphere, 65, pp. 1087-1093, 2006. 